Aims. To establish the improvements in the quality of seclusion medical review after introducing a template to complete the review.

Background. The Mental Health Act - Code of Practice outlines the standards of patient care while in seclusion. It also emphasis that supportive engagement/observation schedules should be reviewed in person and continued at the point an episode of seclusion was initiated.

Furthermore, NICE also set up standards to monitor side effect profile while prescribing psychotropic for such patients and regular management review. It also gives importance to staff training to ensure these standards.

To improve the quality of the seclusion medical review, we completed an audit in July 2019 to ascertain whether medics are following Trust Policy.

We identified good results (above 90\%) in the following areas:

Time of seclusion review

Record keeping

Management plan

Good documentation of risk, mental state examination and physical health.

We also noticed that the following areas can be improved:

Prescribed Medications. (60\%)

Medication side effects. (40\%)

Physical Observations (40\%)

We used the following audit standards for our audit after our last audit and a template was designed and after discussion with medics incorporated into the existing documentation template.

Time of review

Reason and duration for seclusion

Psychiatric diagnosis

Mental State Examination/Behaviour

Physical health (including physical observations)/Environment

Medication (prescribed, rapid tranquilisation, side effects, or adverse effects)

Risk (to self-DSH or accidental) (risks to others)

Plan :(frequency of physical obs./medical review, management, restrictions, exit plan for terminating seclusion, patient's capacity to understand it)

Method. We considered the following aspects:

Retrospective data collection from 01.03.2020 to 30.08.2020.

Sample selection: random selection of mixture of clinicians on different times and days of the week.

Data analysis was carried out by using Microsoft Excel.

Result. We noticed a marked improvement in the quality of seclusion medical review (between 95\% and 100\%) after introducing a template for it. There were no major concerns identified during the re-audit.

Conclusion. To continue to use the template for Seclusion Medical Review which has shown significant improvement in the quality of the reviews which will improve patient care.

It also helped us to deliver person centred care and safe practice.

To continue teaching and training of doctors.

This QIP project motivated nurses to do an audit on nursing seclusion review and made necessary changes.

\section{Quality of seclusion medical review according to trust guidelines}

Shumaila Shahbaz ${ }^{*}$ and Richard Ward

Humber Teaching Foundation Trust

${ }^{*}$ Corresponding author.

doi: 10.1192/bjo.2021.585
Aims. We accessed whether medics are following Trust Policy while conducting seclusion medical review and identify the strengths in quality of seclusion medical review and identify the areas which need improvements to improve our quality and standards of patient's care and safety and to reduce risks.

Background. The Mental Health Act Code of Practice sets an expectation for mental health services for restrictive interventions (use of restraint, seclusion and rapid tranquilisation) by following good standards. Medical reviews provide an opportunity to evaluate and amend seclusion management plan. This clinical audit was undertaken by looking at quality of record keeping about seclusion review by junior doctors, staff grades and consultants at different times (day, night, and weekend).

Method. Data analysis was carried out by using Microsoft Excel. The audit had Humber Teaching NHSFT approval. We assessed electronic healthcare records. Data collection was carried out or retrospectively in 2019( $\mathrm{n}=40)$ using following parameters:

1) A review of patient's physical and psychiatric health.

2) An assessment medication prescribed and adverse effects of medication.

3) A review of observations required.

4) An assessment of the risk posed by the patient to others.

5) An assessment of any risk to the patient from deliberate or accidental self-harm.

6) An assessment of need for continuing seclusion, and whether it is possible for seclusion measures to be applied more flexibly, or in a less restrictive manner.

7) Time of Seclusion Review: Within first hour after seclusion and then every 4 hours until internal MDT. After MDT twice a day.

8) Record Keeping.

Result. Key Successes (above 80\%)

Time of seclusion review (with in first hour or when required)

Record keeping (accurate time and place for clinical notes).

Plan for continuing need for seclusion.

Good documentation of Risk to self and risk to others.

Good documentation of mental state examination.

Comments on physical health although it can be improved.

Key Concerns(Less than 60\%):

Prescribed Medications.

Medication side effects.

Physical Observations

Conclusion. Medics are missing some important parts in seclusion medical review. We developed a template for seclusion medical review according to trust guidelines which are based on Code of Practice and to incorporate in already existing seclusion review form. We also delivered teaching and training to doctors and also showed junior doctor's an example of documentation. We will re-audit in 1 years' time to see improvement.

Pilot project: easy read psychiatry clinic appointment outcome letters

Anu Sharma ${ }^{1 \star}$ and Indermeet Sawhney ${ }^{2}$

${ }^{1}$ Saffron Ground, Ditchmore lane and ${ }^{2}$ Tekhnicon House,

Springwood Drive

${ }^{\star}$ Corresponding author.

doi: 10.1192/bjo.2021.586

Aims. To improve communication with patients and carers by sharing information in an easily comprehensible manner. 